\title{
ISOGEOMETRIC IMPLEMENTATION OF THE HIGH-ORDER MICROPLANE MODEL FOR SOFTENING AND LOCALIZATION
}

\author{
E. LALE* ${ }^{*}$, G. CUSATIS ${ }^{\dagger}$ \\ *Istanbul Technical University \\ Istanbul, Turkey \\ e-mail: lale@itu.edu.tr \\ ${ }^{\dagger}$ Northwestern University \\ Evanston, USA \\ e-mail: g-cusatis@ northwestern.edu
}

Key words: Microplane, high-order continuum, isogeometric, softening, nonlocal model, localization

\begin{abstract}
In this study, a recently developed higher order microplane (HOM) model for softening and localization, is numerically implemented within a finite element framework based on isogeometric analysis. The HOM model was derived directly from a three dimensional discrete particle model and it was shown to be associated with a high order continuum characterized by independent rotation and displacement fields. Furthermore HOM model was demonstrated to possess two characteristic lengths: the first associated with the spacing of flaws in the material internal structure and related to the gradient character of the continuum; and the second associated with the size of these flaws and related to the micropolar character of the continuum. The displacement-based finite element implementation of this type of continua requires $C^{1}$-continuity both within the elements and at the element boundaries. This motivated the implementation of the concept of isogeometric analysis which ensures a higher degree of smoothness and continuity. NURBS based isogeometric elements were implemented in a 3D setting and with both displacement and rotational degrees of freedom at each control point. The performed numerical analyses demonstrate the effectiveness of the proposed HOM model implementation to ensure optimal convergence in both elastic and softening regime. Particularly, the proposed approach prevents strain localization and spurious mesh sensitivity known to be pathological issues for typical local strain-softening constitutive equations.
\end{abstract}

\section{INTRODUCTION}

Behavior of quasi-brittle materials such as concrete, rock, ceramics, cementitous composites and etc., is governed by weak spots of their internal structures which cause initiation of cracks and localization of damage. In order to model this kind of materials' behavior, one can use discrete particle models or continuum models. Each type of model has its own advantages and disadvantages.
For discrete particle models, internal structures of materials are created a priori. They have the advantage that all microscopic effects can be accounted in a straightforward manner. However, large specimens require huge amount of computational resources, which makes these models unfavorable for modeling real size structural elements. On the other hand continuum models are computationally less expensive. But most of the continuum models are based on the Cauchy continuum which can- 
not take into account internal structure. These type of models are not able to produce a mathematically well-posed problem in the softening regime where it cause a strong mesh sensitivity in the numerical results [1,3].

In order to overcome these drawbacks, nonlocal continuum theories are developed. One of the main approaches in these fields is enriching continua with high order strain and stresses. Cosserat brothers study [4] was one of the primary attempts in this field. They considered that material has also rotational degrees of freedom in addition to translational ones. Later many researchers contribute to development of this type of material models. On the other hand, without enriching elastic part, integral or gradient type non-local approaches are also developed and widely used in literature. PijaudierCabot and Bazant paper was one of the pioneering work on this area [13].

Recently Cusatis and Zhou [5], starting from a discrete particle model, derived a high order microplane (HOM) theory which includes Cosserat theory and strain gradients elasticity as a special case. The governing equations of the discrete particle system are homogenized and high order continuum is obtained. HOM model possess two characteristic lengths: the first associated with the spacing of flaws in the material internal structure and related to the gradient character of the continuum; and the second associated with the size of these flaws and related to the micro-polar character of the continuum..

There are some studies on finite element implementation of high-order continuum in the literature. These studies can be separated into two categories: $C^{1}$ continuity implementation and mixed element implementation. The displacement-based finite element implementation of this type of continua requires $C^{1}$ continuity both within the elements and at the element boundaries. Papanicolopulos et al. [11], Zervos et. al. [16] developed $C^{1}$ type finite element for gradient elasticity. In addition Fischear et al implemented isogeometric analysis in 2D case for gradient elasticity [7]. On the other hand, in mixed formulations $C^{0}$ con- tinuity element can be used but in this case first derivatives should be interpolated in addition to displacements and these types of elements are required to fulfill some patch tests.

In this study displacement based finite element implementation of high-order microplane model is conducted by employing isogeometric analysis concept. NURBS based isogeometric elements were implemented in a 3D setting and with both displacement and rotational degrees of freedom at each control point.

\section{HIGHER ORDER MICROPLANE MODEL}

If we consider a represented volume composed of particles and assume that deformation of particles are negligible and damage is localizing at the contact point (microplane), then deformations can be calculated in terms of relative displacement and rotations of particles as follows:

(a)

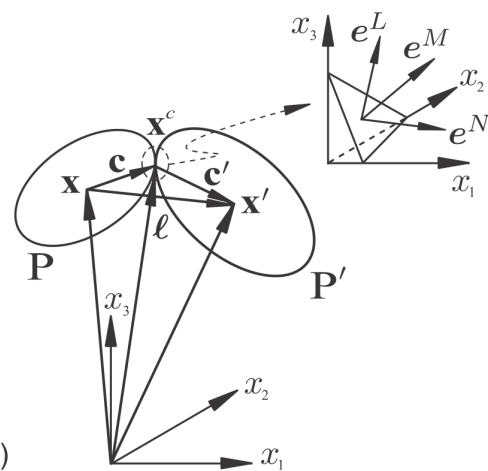

(b)

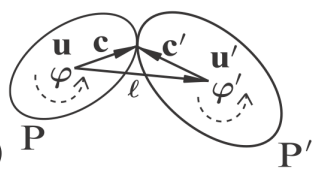

Figure 1: Geometry description of two adjacent particles in contact.

$$
\mathbf{s}=l^{-1}[\Delta \mathbf{u}-\varphi \times \mathbf{l}-\Delta \varphi \times(\mathbf{l}-\mathbf{c})]
$$

where $\Delta \mathbf{u}=\mathbf{u}^{\prime}-\mathbf{u}$ and $\Delta \varphi=\varphi^{\prime}-\varphi, \mathrm{u}$ and $\varphi$ is displacement and rotation vector of particles. 1 represents the vector connecting adjacent particle centroid and $\mathbf{c}$ is the vector connecting par- 
ticle centroid to weak point located at the contact point. With reference to Fig 1, normal and tangential component of strains and curvatures can be obtained using direction cosines as follows:

$$
\varepsilon_{\beta}=l^{-1} e_{i}^{\beta} \Delta u_{i}-\epsilon_{i j k} e_{i}^{\beta} \varphi_{j} e_{k}^{l}-\epsilon_{i j k} e_{i}^{\beta} \Delta \varphi_{j}\left(e_{k}^{l}-\alpha e_{k}^{c}\right)
$$

$$
\chi_{\beta}=l^{-1} \Delta \varphi_{i} e_{i}^{\beta}
$$

where $\beta$ denotes microplane directions and $\alpha=c / l$. If we expand the displacement and rotations fields in Taylor series around centroid of represented volume and omit higher order term then displacement and rotation jump can be estimated as follows,

$$
\begin{gathered}
\Delta u_{i}=l\left[u_{i, j}^{0} e_{j}^{l}+u_{i, j k}^{0}\left(x_{j} e_{k}^{l} / 2+x_{k} e_{j}^{l}+l e_{j}^{l} e_{k}^{l}\right)\right] \\
\Delta \varphi_{i}=l \varphi_{i, j}^{0} e_{j}^{l}
\end{gathered}
$$

Substituting these terms into Equation 2-3 and after some manipulation microplane deformations and curvatures read:

$$
\begin{gathered}
\varepsilon_{\beta}=N_{i j}^{\beta} \gamma_{i j}+\frac{l}{2} N_{i j k}^{\beta} \Gamma_{i j k}+\frac{l}{2} P_{i j}^{\beta} \kappa_{i j} \\
\chi_{\beta}=N_{i j}^{\beta} \kappa_{i j}
\end{gathered}
$$

where $\gamma_{i j}=u_{j, i}^{0}-\epsilon_{i j k} \varphi_{k}^{0}$ is strain tensor, $\kappa_{i j}=$ $\varphi_{j, i}^{0}$ is curvature tensor and $\Gamma_{i j k}=\gamma_{i j, k}$ is strain gradient tensor (high order strain). Furthermore if we assume that all particles are sphere and their size is equal then microplane strains can be simplified as follows $\left(l=l_{0}=2 r_{0}\right.$, $\left.e_{i}^{l}=e_{i}^{c}=e_{i}^{N}, \alpha=1 / 2, P_{i j}^{\beta}=0\right)$ :

$$
\varepsilon_{\beta}=N_{i j}^{\beta} \gamma_{i j}+r_{0} N_{i j k}^{\beta} \Gamma_{i j k}
$$

Here $N_{i j}=e_{i}^{N} e_{j}^{\beta}$ and $N_{i j k}=e_{i}^{N} e_{j}^{\beta} e_{k}^{N}$ denote projection operators for strain and strain gradient respectively.

Using appropriate vectorial constitutive model, microplane stresses and couple stresses can be calculated. After calculation of microplane stresses, macroscopic stress and highorder stress are obtained by applying virtual work theory over a spherical domain $\Omega_{0}[2,5]$

$$
\begin{aligned}
\sigma_{i j} & =\frac{3}{4 \pi} \int_{\Gamma_{1}} \sigma_{\beta} N_{i j}^{\beta} d s \\
\mu_{i j} & =\frac{3}{4 \pi} \int_{\Gamma_{1}} \mu_{\beta} N_{i j}^{\beta} d s \\
\Sigma_{i j k} & =\frac{3 r_{0}}{4 \pi} \int_{\Gamma_{1}} \sigma_{\beta} N_{i j k}^{\beta} d s
\end{aligned}
$$

where the integrals are calculated over the surface of the unit sphere, $\Gamma_{1}$.

For completeness of constitutive model, equilibrium equation is summarized here. If one employs virtual work theory, equilibrium equation is obtained as follows [5]:

$$
\pi_{j i, j}+b_{i}=0 ; \quad \mu_{j i, j}+\epsilon_{i j k} \pi_{j k}+a_{i}=0
$$

where $a_{i}$ and $b_{i}$ denote body forces and moments, $\epsilon_{i j k}$ is permutation symbol and $\pi_{i j}$ is effective stress defined as follows

$$
\pi_{i j}=\sigma_{i j}-\Sigma_{i j k, k}
$$

Similarly boundary conditions can be obtained too, detailed information can be found in [5].

In order to investigate localization properties of the proposed model, a spectral analysis is conducted for $1 \mathrm{D}$ case where $u=u(x)$, $\epsilon=u_{, x}+r_{0} u_{, x x}$ and $\psi=r_{0} u_{x x}$ can be written. Then effective stress reads:

$$
\pi=\sigma(\epsilon)-r_{0} \sigma(\epsilon)_{, x}-r_{0} H_{0} \psi_{, x}
$$

In the absence of body forces, $\pi$ must be constant along $\mathrm{x}$ direction. Considering linear softening with modulus $H_{t}, \sigma=\sigma_{0}-H_{t}\left(\epsilon-\epsilon_{0}\right)$, one can obtain phase velocity as below:

$$
c=\sqrt{\left[-H_{t}+r_{0}^{2} k^{2}\left(H_{0}-H_{t}\right)\right] / \rho}
$$

Which clearly indicate that phase velocity is imaginary for any wave length if the regularization term, $H_{0}$, is set to zero. On the other hand, with proper selection of regularization parameter, one can get a real phase velocity. If localization band is assumed to equal to $2 r_{0}$ then $H_{0}$ reads 


$$
H_{0}=H_{t}\left(1+1 / \pi^{2}\right)
$$

In ref. [5] regularization parameter is given by $\psi_{\beta}=r_{0} N_{i j k}^{\beta} \Gamma_{i j k}$. In fact $\psi$ is the contribution of higher order terms on microplane strains and multiplication of it with $H_{0}$ gives a kind of microplane stresses:

$p_{\beta}=H_{0} \psi_{\beta}=H_{t} \psi_{\beta}\left(1+1 / \pi^{2}\right)=\sigma_{\beta}^{h o}\left(1+1 / \pi^{2}\right)$

Here $\sigma_{\beta}^{\text {ho }}=H_{t} \psi_{\beta}$ is the increment of microplane stresses due to higher order terms. Numerical implementation shows that this type of regularization produce more ductile softening when mesh size is getting smaller which means that it is not realistic. Because of this reason and with considering incremental form of nonlinear equation solver, regularization term is assumed as the negative value of microplane stress increment, $\Delta p_{\beta}=\Delta \sigma_{\beta}\left(1+1 / \pi^{2}\right)$, and high-order stresses are reformulated as follows:

$$
\Delta \Sigma_{i j k}^{n}=\frac{3 r_{0}}{4 \pi} \int_{\Gamma_{1}}\left(\Delta \sigma_{\beta}^{n}+\Delta p_{\beta}\right) N_{i j k}^{\beta} d s
$$

\section{FINITE ELEMENT IMPLEMENTA- TION}

\subsection{Concept of Isogeometric Analysis}

Isogeometric analysis is introduced by Hughes et al. It combines computer aided design (CAD) and computer aided analysis technology [8]. CAD technology commonly employ NURBS basis functions. NURBS means Non-Uniform Rational B-Splines and they are built from B-splines. A B-spline curve is a piecewise polynomial composed of a linear combination of basis functions as follows,

$$
C(\xi)=\sum_{i=1}^{n} N_{i, p}(\xi) B_{i}
$$

where $p$ and $n$ denote the order and number of the basis functions, $B_{i}$ is control points and $N_{i, p}$ is basis function. B-spline basis functions are linearly independent, have partition of unity property and their support is compact. But they don't satisfy Kronecker delta property which prevents application of boundary condition directly on control points $[8,10]$. B-splines basis are defined over a knot vector, $\Xi$, which is a set of non-decreasing real numbers representing coordinates in the parameter domain:

$$
\Xi=\left\{\xi_{1}, \xi_{2}, \ldots, \xi_{n+p+1}\right\}
$$

A B-spline is said to be open if its first and last knots appear $p+1$ times. Open knot vectors are interpolatory at the end points which mean they satisfy Kronecker delta property at those points.

B-spline basis functions are defined recursively starting with piece-wise constants $(p=$ $0)$ :

$$
N_{i, 0}=\left\{\begin{array}{ll}
1 & \xi_{i} \leq \xi<\xi_{i+1} \\
0 & \text { otherwise. }
\end{array}\right\}
$$

and higher order basis functions $(p>0)$ are defined in terms of lower order basis as below: $N_{i, p}(\xi)=\frac{\xi-\xi_{i}}{\xi_{i+p}-\xi_{i}} N_{i, p-1}(\xi)+\frac{\xi_{i+p+1}-\xi}{\xi_{i+p+1}-\xi_{i+1}} N_{i+1, p-1}(\xi)$

NURBS base functions are defined in terms of B-spline basis as follows,

$$
R_{i, p}(\xi)=\frac{N_{i, p}(\xi)}{W(\xi)}=\frac{N_{i, p}(\xi)}{\sum_{j=1}^{n} N_{j, p}(\xi) w_{j}}
$$

where $w_{j}$ represent weighting factor and many different types of curves can be defined by selecting appropriate value for the weight. Bspline basis are the special case of the NURBS basis when the all control points have the same weight. A NURBS curve is defined in a similar manner with B-Spline curves as given :

$$
C(\xi)=\sum_{i=1}^{n} R_{i, p}(\xi) B_{i}
$$

\subsection{Implementation of isogeometric ele- ment}

Isogeometric element is implemented in 3D and displacement field and rotational field is assumed independent. It has totally 6 degrees of freedom in each control points which consist of 3 displacement and 3 rotations. Following the isoparametric concept, the displacement field 
$u$ and rotation field $\varphi$ is formulated using the same NURBS basis functions with the geometry:

$$
u=\sum_{i=1}^{n} R_{i} \mathbf{u}_{i}^{e} \quad \Phi=\sum_{i=1}^{n} R_{i} \boldsymbol{\varphi}_{i}^{e}
$$

where $\mathbf{u}^{e}=\left[u_{x i}, u_{y i}, u_{z i}\right]$ denotes nodal displacement vector and $\varphi^{e}=\left[\varphi_{x i}, \varphi_{y i}, \varphi_{z i}\right]$ is nodal rotation vector. If $\mathbf{e}=\left[\gamma_{i j}, \kappa_{i j}, \Gamma_{i j k}\right]$ is strain vector and $\mathbf{d}=\left[u_{i}, \phi_{i}\right]$ is nodal displacement vector then following equation can be written:

$$
\mathbf{e}=\mathbf{B d}
$$

where $\mathbf{B}$ denote strain displacement matrix and it is obtained applying differential operator $\mathbf{L}$ on shape (basis) functions, $\mathbf{B}=\mathbf{L R}$. Once the strain displacement matrix, $\mathbf{B}$, is obtained element stiffness matrix, internal force and mass matrix can be calculated easily by employing appropriate quadrature rule.

$$
K=\int_{\Omega} \mathbf{B}^{T} \mathbf{D B} \mathrm{d} \Omega, \quad f=\int_{\Omega} \mathbf{B}^{T} \sigma \mathrm{d} \Omega
$$

\section{NUMERICAL RESULTS}

\subsection{Elastic cantilever beam}

Result of isogeometric finite element with high-order strain is presented in this section. First a cantilever beam, $1000 \mathrm{~mm}$ long with $100 \times 25 \mathrm{~mm}$ cross-section, is modeled with quadratic isogeometric solid elements. Beam is discretized with 4 different meshes, $10 \times 1 \times 1$, $20 \times 2 \times 1,40 \times 4 \times 1,80 \times 8 \times 2$. All boundary condition assumed as essential so load is applied by imposing displacement on the free end of the beam. In Fig. 2 2 relative displacement (normalized with value obtained using Cauchy Continuum) versus non-dimensional curves of beam with $l_{c}=0.2 \mathrm{~L}$ are given for different meshes with analytical result given by Beskou et al [12]. As can be seen from the figure, with mesh refinement tip displacement of isogeometric element solution converges to a value. But for this case elastic curve deviate from analytical solution. Main reason is that in analytial solution, they only consider one high-order term
$\Gamma_{x x x}=\gamma_{x x, x}$, but proposed HOM model is a 3D model and it takes into account other terms too. In addition, it should be kept in mind that this amount of length scale is chosen from the mathematical point of view, but physically it is not realistic. In fact characteristic length value can be in the order of 0.1-0.2 times of the crosssectional dimensions of a beam.

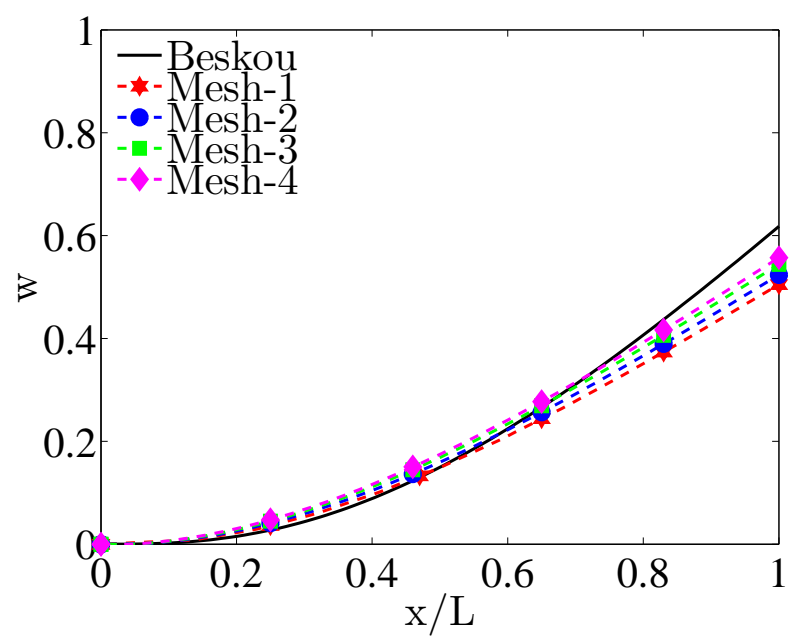

Figure 2: Relative displacement $w$ versus x curve for different mesh $\left(l_{c}=0.2 L\right)$.

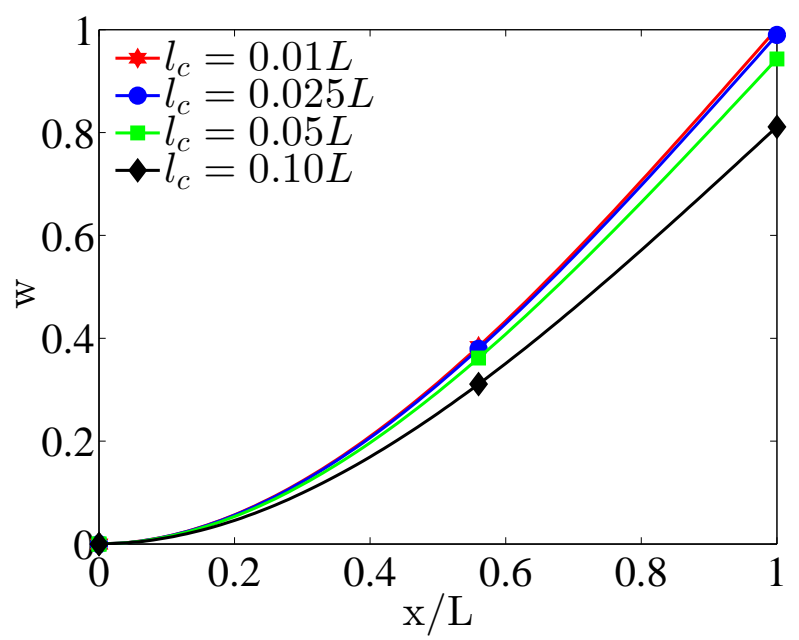

Figure 3: Relative displacement $w$ versus x curve for different internal length scale

Fig. 3 shows the obtained curves of relative displacement versus non-dimensional coordinates for different internal length scale. Three different internal length scales, $l_{c}=$ 
$0.1 L, 0.05 L, 0.025 L, 0.01 L$ are used. As expected with increase of internal length scale, beam becomes stiffer. For lower internal length to beam length ratio, response converge to classical beam solution. Especially $l_{c}$ is smaller than $0.01 \mathrm{~L}$, results are almost identical to Cauchy solution. For higher $l_{c} / L$ ratio, discrepancy from Cauchy solution becomes obvious.

\subsection{Plate with a hole}

In order to demonstrate the efficiency of the isogeometric element for high-order continuum theory, a well-known problem, an infinite plate with a hole subjected to a one way far field tension load is modeled. Analytical solutions of this problem for classical Cosserat continuum was given by Eringen [6] and for Couple Stress theory by Mindlin [9] in 2D case.

This example was also simulated using FEM by Pothier and Rencis [14] and Nakamura et. al. [15]. Same parameter are used for comparison and they are reported in Table 11. Plate dimensions are considered as $300 \times 300 \times 10 \mathrm{~mm}$ and the hole radius is $r=10 \mathrm{~mm}$. Considering the symmetry along $\mathrm{x}$ - and $\mathrm{y}$-axes, only one quarter of the specimen is modeled. A 3D finite element model is set up with quadratic isogeometric solid element. Two different mesh is used which has $1024(32 \times 32)$ and 4096 $(64 \times 64)$ elements. For both mesh only one element is used along the thickness.

Table 1: Material parameters for plate with a hole

\begin{tabular}{clllll}
\hline Case & $N_{c}$ & $\mathrm{E}$ & $\nu$ & $\chi$ & $\pi_{2}$ \\
\hline S1 & 0.00 & $1.733 \mathrm{E} 4$ & 0.300 & 0.0 & $1.71 \mathrm{E} 8$ \\
\hline S2 & 0.25 & $1.832 \mathrm{E} 4$ & 0.308 & $1.0 \mathrm{E} 3$ & $1.92 \mathrm{E} 9$ \\
\hline S3 & 0.50 & $2.692 \mathrm{E} 3$ & 0.346 & $1.0 \mathrm{E} 3$ & $3.84 \mathrm{E} 8$ \\
\hline S4 & 0.70 & $2.692 \mathrm{E} 3$ & 0.346 & $5.0 \mathrm{E} 4$ & $3.84 \mathrm{E} 8$ \\
\hline
\end{tabular}

In numerical simulations, higher order terms are neglected by assuming associated material characteristic length equals to zero, $r_{0}=0$, in order to make one to one equivalence of Cosserat continuum given by Eringen with proposed HOM theory. Furthermore relations be- tween microplane material stiffness and classical Cosserat elasticity parameters should be taken as given in Ref. [5].

The comparison of the stress concentration factors obtained by simulation with analytic solution, is presented in Table 2. Results show very good agreement with analytical values. Furthermore effect of coupling factor on the SIF can be obtained properly.

Table 2: Stress intensity factor for elastic plate

\begin{tabular}{lccc}
\hline Case & Analytical & Mesh-1 & Mesh-2 \\
\hline SIM1 & 3.0 & 2.894 & 2.999 \\
SIM2 & 2.839 & 2.735 & 2.813 \\
SIM3 & 2.482 & 2.436 & 2.474 \\
SIM4 & 2.034 & 2.011 & 2.034 \\
\hline
\end{tabular}

\subsection{Softening of a bar under uniaxial ten- sion}

In order to assess regularization capability of the proposed model, a prismatic bar subjected to uniaxial tension is considered. It is modeled in 3D with quadratic isogeometric solid elements. Couple stresses and micro curvatures are neglected in this example. The bar is 100 $\mathrm{mm}$ long and has $75 \times 75 \mathrm{~mm}$ rectangular cross section. One end of the bar is fixed in all directions and displacement is prescribed opposite end in the longitudinal direction.

Material characteristic length is assumed, $r_{0}=5 \mathrm{~mm}$, and four different meshes are used $10 \times 1 \times 1,20 \times 1 \times 1,40 \times 1 \times 1$ and $80 \times 1 \times 1$. Selected material parameters are Young modulus $E=25000 \mathrm{MPa}$, Poisson ratio $\nu=0.2$ and tensile strength $3 \mathrm{MPa}$. Stress integration is performed by a Voronoi integration rule with 66 microplane over a whole unit sphere. In order to fix the localization zone, center part (one tenth) of the bar is weakened by reducing the tensile strength 10 percent. 


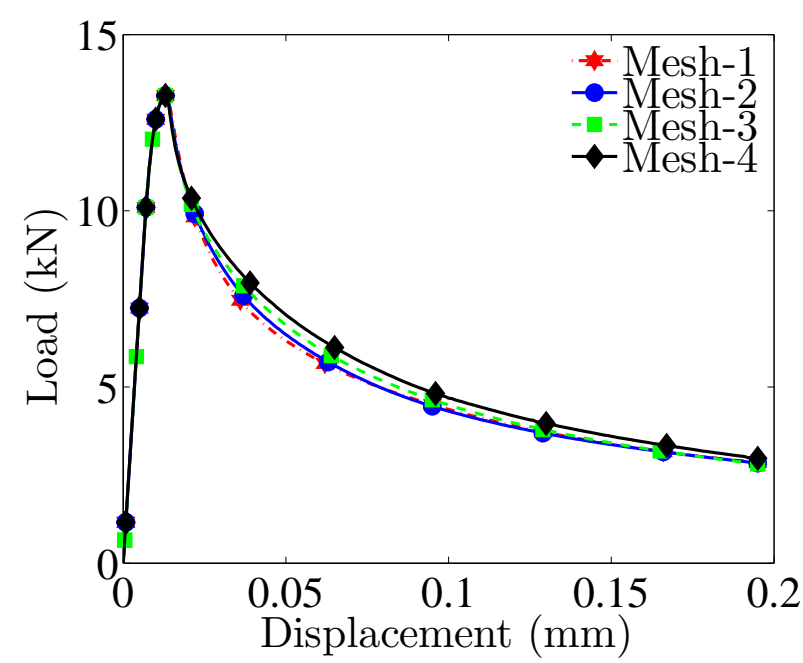

Figure 4: Load-displacement curve for bar under uniaxial tension

Obtained load-displacement is given in Fig. 4. It can be observed that the solution converges properly as the number of elements increase. Strain profile along longitudinal axis of the bar is also demonstrated in Fig. 5, as can be seen from this figure the width of the localization zone almost doesn't change and maximum strain value converges with mesh refinement which means it is independent from the element size. These results clearly indicate that proposed regularization technique is able to prevent mesh dependency.

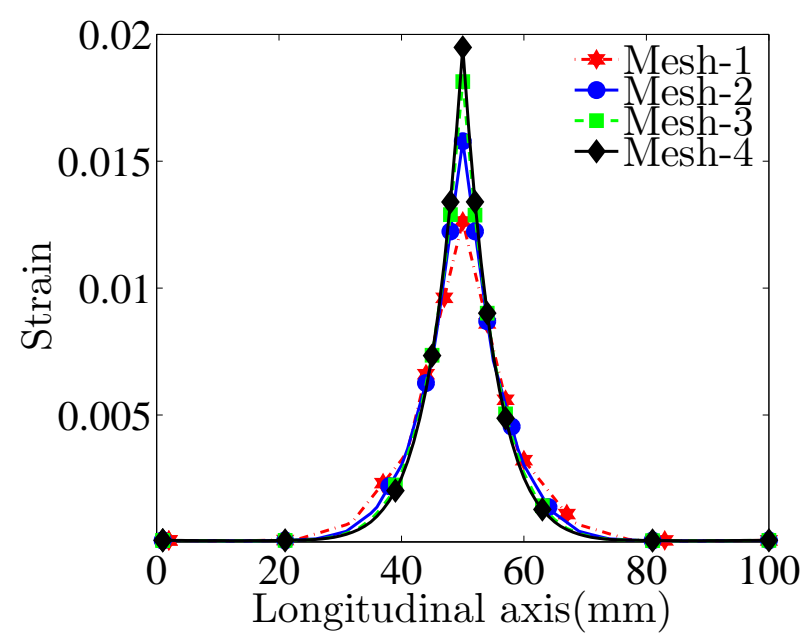

Figure 5: Strain profile along the bar for regularized case

\section{CONCLUSIONS}

In this study isogeometric finite element implementation of high-order microplane model is introduced. HOM model was derived based on discrete particle model in order to mimic it as a continuum. Model produces a generalized media which includes gradient elasticity and Cosserat theory as a special case. Firstly, validation of the model in elastic case is presented by comparing with analytical solution given in literature for Cosserat continuum and Gradient elasticity. Then performance of highorder microplane model and proposed regularization method for softening is given for a bar subjected to uniaxal tension. Proposed regularization method gives excellent result which prevents localization and spurious mesh dependency is completely removed.

\section{REFERENCES}

[1] Zdenek P Bazant, Ted B Belytschko, and Ta-Peng Chang. Continuum theory for strain-softening. Journal of Engineering Mechanics, 110(12):1666-1692, 1984.

[2] Zdenek P Bazant, Ferhun C Caner, Ignacio Carol, Mark D Adley, and Stephen A Akers. Microplane model m4 for concrete. i: Formulation with work-conjugate deviatoric stress. Journal of Engineering Mechanics, 126(9):944-953, 2000.

[3] Zdenek P Bazant and Gilles PijaudierCabot. Nonlocal continuum damage, localization instability and convergence. Journal of applied mechanics, 55(2):287293, 1988.

[4] Eugène Cosserat and François Cosserat. Théorie des corps déformables. Paris, 1909.

[5] Gianluca Cusatis and Xinwei Zhou. High-order microplane theory for quasibrittle materials with multiple characteristic lengths. Journal of Engineering Mechanics, 2013. 
[6] AC Eringen. Theory of micropolar elasticity. Technical report, DTIC Document, 1967.

[7] Paul Fischer, Markus Klassen, Julia Mergheim, Paul Steinmann, and Ralf Müller. Isogeometric analysis of $2 \mathrm{~d}$ gradient elasticity. Computational Mechanics, 47(3):325-334, 2011.

[8] Thomas JR Hughes, John A Cottrell, and Yuri Bazilevs. Isogeometric analysis: Cad, finite elements, nurbs, exact geometry and mesh refinement. Computer methods in applied mechanics and engineering, 194(39):4135-4195, 2005.

[9] RD Mindlin. Influence of couple-stresses on stress concentrations. Experimental mechanics, 3(1):1-7, 1963.

[10] Vinh Phu Nguyen, Stéphane Bordas, and Timon Rabczuk. Isogeometric analysis: an overview and computer implementation aspects. arXiv preprint arXiv:1205.2129, 2012.

[11] S-A Papanicolopulos, A Zervos, and I Vardoulakis. A three-dimensional c1 finite element for gradient elasticity. International journal for numerical methods in engineering, 77(10):1396-1415, 2009.
[12] S Papargyri-Beskou, KG Tsepoura, D Polyzos, and DE Beskos. Bending and stability analysis of gradient elastic beams. International Journal of Solids and Structures, 40(2):385-400, 2003.

[13] Gilles Pijaudier-Cabot and Zdenek P Bazant. Nonlocal damage theory. Journal of engineering mechanics, 113(10):15121533, 1987.

[14] A Pothier and JJ Rencis. Threedimensional finite element formulation for microelastic solids. Computers \& structures, 51(1):1-21, 1994.

[15] Nakamura Sachio, Robert Benedict, and Roderic Lakes. Finite element method for orthotropic micropolar elasticity. International Journal of Engineering Science, 22(3):319-330, 1984

[16] A Zervos, P Papanastasiou, and I Vardoulakis. A finite element displacement formulation for gradient elastoplasticity. Bifurcation and Localisation Theory in Geomechanics (H. B. Mühlhaus, A. Dyskin and E. Pasternak, eds.), Balkema, pages 177-187, 2001. 